\title{
WEIGHTED NORM INEQUALITIES FOR THE GEOMETRIC MAXIMAL OPERATOR
}

\author{
David Cruz-Uribe, SFO, and C. J. Neugebauer
}

Abstract

We consider two closely related but distinct operators,

$$
\begin{aligned}
& M_{0} f(x)=\sup _{I \ni x} \exp \left(\frac{1}{|I|} \int_{I} \log |f| d y\right) \text { and } \\
& M_{0}^{*} f(x)=\lim _{r \rightarrow 0} \sup _{I \ni x}\left(\frac{1}{|I|} \int_{I}|f|^{r} d y\right)^{1 / r}
\end{aligned}
$$

We give sufficient conditions for the two operators to be equal and show that these conditions are sharp. We also prove twoweight, weighted norm inequalities for both operators using our earlier results about weighted norm inequalities for the minimal operator:

$$
m f(x)=\inf _{I \ni x} \frac{1}{|I|} \int_{I}|f| d y .
$$

This extends the work of X. Shi; H. Wei, S. Xianliang and S. Qiyu; X. Yin and B. Muckenhoupt; and C. Sbordone and I. Wik.

\section{Introduction}

Given a real-valued, measurable function $f$ on $\mathbb{R}^{n}$, the geometric maximal function of $f$ is

$$
M_{0} f(x)=\sup _{I} \exp \left(\frac{1}{|I|} \int_{I} \log |f| d y\right),
$$

Keywords. Maximal operator, minimal operator, weighted norm inequalities. 1991 Mathematics subject classifications: 42B25. 
where the supremum is taken over all cubes $I$ which contain $x$ and whose sides are parallel to the co-ordinate axes. Closely related to the geometric maximal operator is the following sequence of maximal operators: for $f$ as before and for any $r>0$ define

$$
M_{r} f(x)=\sup _{I}\left(\frac{1}{|I|} \int_{I}|f|^{r} d y\right)^{1 / r}
$$

where the supremum is again taken over all cubes containing $x$. Equivalently, $M_{r} f=M\left(f^{r}\right)^{1 / r}$, where $M$ is the Hardy-Littlewood maximal operator. By Hölder's inequality, for $s<r, M_{s} f(x) \leq M_{r} f(x)$, so we may define the limiting operator $M_{0}^{*}$ by

$$
M_{0}^{*} f(x)=\lim _{r \rightarrow 0} M_{r} f(x) .
$$

By Jensen's inequality, $M_{0} f(x) \leq M_{0}^{*} f(x)$. Since we have the wellknown limit

$$
\lim _{r \rightarrow 0}\left(\frac{1}{|I|} \int_{I}|f|^{r} d y\right)^{1 / r}=\exp \left(\frac{1}{|I|} \int_{I} \log |f| d y\right)
$$

(see Rudin [9, p. 74]), it is reasonable to conjecture that for all functions $f$ such that for some $r>0, f^{r} \in L_{\text {loc }}^{1}, M_{0}^{*} f(x)=M_{0} f(x)$ a.e. However, as we will show below, this is not true in general.

The purpose of this paper is to study the relation between $M_{0}$ and $M_{0}^{*}$, and to prove two-weight, weighted norm inequalities for each operator. These problems have been considered previously, with mixed results. In 1980, X. Shi [11] proved the following one-weight norm inequality.

Theorem 1.1. Given a weight $w$, the following are equivalent:

1. $w \in A_{\infty}$ : there exists a constant $C$ such that for all cubes $I$,

$$
\frac{1}{|I|} \int_{I} w d x \leq C \exp \left(\frac{1}{|I|} \int_{I} \log w d x\right) .
$$

2. For $0<p<\infty$ the strong-type norm inequality

$$
\int_{\mathbb{R}^{n}}\left(M_{0} f\right)^{p} w d x \leq C \int_{\mathbb{R}^{n}}|f|^{p} w d x
$$

holds for all $f \in L^{p}(w)$. 
(The equivalence of the $A_{\infty}$ condition and the so-called reverse Jensen inequality was not apparently discovered by Shi; it was discovered independently by García-Cuerva and Rubio de Francia [6] and Hrusčev [7].)

In 1991, H. Wei, S. Xianliang and S. Qiyu [12] attempted to extend this result to the two-weight case on spaces of homogeneous type. Their proof, however, contained an error. This was pointed out by X. Yin and B. Muckenhoupt $[\mathbf{1 3}]$, who proved the following pair of two-weight norm inequalities on $\mathbb{R}^{1}$.

Theorem 1.2. Given a pair of weights $(u, v)$, the following are equivalent:

1. $(u, v) \in W_{\infty}$ : there exists a constant $C$ such that for all intervals $I$

$$
\frac{1}{|I|} \int_{I} u d x \leq C \exp \left(\frac{1}{|I|} \int_{I} \log v d x\right) .
$$

2. For $0<p<\infty$ the weak-type norm inequality

$$
u\left(\left\{x: M_{0} f(x)>t\right\}\right) \leq \frac{C}{t^{p}} \int_{\mathbb{R}}|f|^{p} v d x
$$

holds for all $f \in L^{p}(v)$.

Theorem 1.3. Given a pair of weights $(u, v)$, the following are equivalent:

1. $(u, v) \in W_{\infty}^{*}$ : there exists a constant $C$ such that for all intervals $I$

$$
\int_{I} M_{0}\left(v^{-1} \chi_{I}\right) u d x \leq C|I| .
$$

2. For $0<p<\infty$ the strong-type norm inequality

$$
\int_{\mathbb{R}}\left(M_{0} f\right)^{p} u d x \leq C \int_{\mathbb{R}}|f|^{p} v d x
$$

holds for all $f \in L^{p}(v)$.

Their proofs depend heavily on covering lemmas which are particular to the real line. Therefore it is doubtful that they can be extended to higher dimensions.

Yin and Muckenhoupt also gave a complicated example to show that the class $W_{\infty}^{*}$ is strictly contained in $W_{\infty}$. (Also note that in the twoweight case the class $W_{\infty}$ is strictly larger than $A_{\infty}=\cup_{p} A_{p}$-a simple example is the pair $\left(e^{|x|}, e^{|2 x|}\right)$.) 
Finally, they assert in passing that $M_{0} f$ and $M_{0}^{*} f$ are the same "for suitably restricted $f$ ". However, they give no indication of what this means.

Independently of these three papers, in 1994 C. Sbordone and I. Wik $[\mathbf{1 0}]$ published a different proof of Theorem 1.1. Their proof, however, requires that for all locally integrable $f, M_{0} f(x)=M_{0}^{*} f(x)$, which is false. There is a simple counter-example: let $C$ be a nowhere dense subset of $[0,1]$ such that $|C|=1 / 2$, and define $f=\chi_{C}$. Then for each interval $I \subset[0,1], \int_{I} \log |f| d x=-\infty$, so $M_{0} f(x) \equiv 0$. But by the Lebesgue differentiation theorem, $M_{r} f(x) \geq f(x)$ for almost every $x \in[0,1]$ and each $r>0$, so $M_{0}^{*} f(x)=1$ on a set of measure one-half. (The error in their proof is in inequality (2.11), as this example shows.)

We prove the following results: in Section 2 we give sufficient conditions on a function $f$ for the equality $M_{0} f(x)=M_{0}^{*} f(x)$ to hold almost everywhere. Our main result shows that for equality to hold $\log f$ must be locally integrable and the size of $f$ at infinity must be controlled.

Theorem 1.4. Given a function $f$ on $\mathbb{R}^{n}$, the equality $M_{0} f(x)=$ $M_{0}^{*} f(x)$ holds for almost every $x$ if one of two conditions holds:

1. $f \in L^{p}\left(\mathbb{R}^{n}\right)$ for some $p, 0<p<\infty$, and $\log f \in L_{\text {loc }}^{1}$;

2. $f \in L^{\infty}\left(\mathbb{R}^{n}\right)$ and for some $\alpha>1, M\left(|\log f|^{\alpha}\right)(x)<\infty$ a.e.

Neither of these conditions is strictly necessary - counter-examples can be readily constructed using monotonically decreasing functions. However, we give examples to show that if either condition is weakened then equality need not hold in general.

In Section 3 we give new proofs of Theorems 1.1, 1.2 and 1.3. Our proofs depend on the weighted norm inequalities for the minimal operator: given a real-valued, measurable function $f$ on $\mathbb{R}^{n}$, the minimal function of $f$ is

$$
m f(x)=\inf _{I} \frac{1}{|I|} \int_{I}|f| d y
$$

where the infimum is taken over all cubes containing $x$. Intuitively, the minimal operator controls where a function is small, just as the maximal operator controls where it is large. We introduced the minimal operator in $[2]$ in order to study the fine structure of functions which satisfy the reverse Hölder inequality. In that paper we also studied the one-weight norm inequalities which it satisfies. In [3], Cruz-Uribe, Neugebauer and Olesen examined the two-weight norm inequalities for the minimal operator on $\mathbb{R}^{1}$. (Additional results about variants of the minimal operator can be found in $[\mathbf{4}]$ and $[5]$.) 
Our approach has two advantages. First, the proofs are considerably simpler, though part of the reason for this is that the work is in the proof of the norm inequalities for the minimal operator. Second, in the two-weight case our proofs extend to higher dimensions, provided that we can characterize the weights governing the norm inequalities for the minimal operator in higher dimensions. We obtained partial results in higher dimensions in [3]: for example, our proof of the two-weight, weak-type norm inequality extends to $\mathbb{R}^{n}$ if we assume that $u$ is doubling. More systematic results which yield sufficient conditions (both with and without doubling conditions) for norm inequalities for $M_{0}$ and $M_{0}^{*}$ will appear in Cruz-Uribe [1].

At the end of Section 3 we give another example (simpler than that of Yin and Muckenhoupt) to show that the class $W_{\infty}^{*}$ is smaller than $W_{\infty}$.

In Section 4 we prove results analogous to Theorems 1.1, 1.2 and 1.3 for $M_{0}^{*}$. In examining this operator, a key difficulty was the fact that there exist functions $f$ such that if $Q_{n}$ is the cube of side $2 n$ centered at the origin, then

$$
\lim _{n \rightarrow \infty} M_{0}^{*}\left(f \chi_{Q_{n}}\right)(x)<M_{0}^{*} f(x)
$$

for $x$ in a set of positive measure. (In other words, we could not a priori restrict ourselves to functions of compact support and then obtain the final result using the monotone convergence theorem.) For example, let $f=1-\chi_{[0,1]}$. Then for all $n>0$ and all $x \in(0,1)$ it is easy to see that $M_{0}^{*}\left(f \chi_{[-n, n]}\right)(x)=0$ while $M_{0}^{*} f(x)=1$.

Initially, we avoided this problem by assuming a growth condition on $v$ : we say that $v$ satisfies the $I_{\infty}$ condition if

$$
\limsup _{I, \sigma} \frac{1}{|I|}\left(\frac{1}{|I|} \int_{I} v^{-\sigma} d x\right)^{1 / \sigma}<\infty,
$$

where the limit supremum is taken over all cubes $I$ containing the origin and all $\sigma>0$ as $|I|$ tends to infinity and as $\sigma$ tends to zero. This condition appears unnatural; however, it is the formal limit as $p$ tends to infinity of the condition

$$
\limsup _{|I| \rightarrow \infty} \frac{1}{|I|}\left(\frac{1}{|I|} \int_{I} v^{-p^{\prime} / p} d x\right)^{p / p^{\prime}}<\infty,
$$

which Rubio de Francia [8] showed is a necessary and sufficient condition on a weight $v$ for there to exist $u$ such that $(u, v)$ is in the Sawyer class $S_{p}$. (This class governs the strong-type norm inequalities for the Hardy-Littlewood maximal operator. For details, see García-Cuerva and Rubio de Francia [6]. We are grateful to A. de la Torre for pointing this relation out to us.) 
By assuming the $I_{\infty}$ condition we were able to reduce first to the case of functions of compact support, and then to the case of functions for which $M_{0}$ and $M_{0}^{*}$ are equal. In this case we could then apply Theorems 1.1, 1.2 and 1.3. To our surprise, we were able to show that the $I_{\infty}$ condition is necessary as well. In $\mathbb{R}^{1}$ we thus proved the following analogues of Theorems 1.2 and 1.3.

Theorem 1.5. Given a pair of weights $(u, v)$, then for $0<p<\infty$ the weak-type norm inequality

$$
u\left(\left\{x: M_{0}^{*} f(x)>t\right\}\right) \leq \frac{C}{t^{p}} \int_{\mathbb{R}}|f|^{p} v d x
$$

holds for all $f \in L^{p}(v)$ if and only if $(u, v) \in W_{\infty}$ and $v \in I_{\infty}$.

Theorem 1.6. Given a pair of weights $(u, v)$, then for $0<p<\infty$ the strong-type norm inequality

$$
\int_{\mathbb{R}}\left(M_{0}^{*} f\right)^{p} u d x \leq C \int_{\mathbb{R}}|f|^{p} v d x
$$

holds for all $f \in L^{p}(v)$ if and only if $(u, v) \in W_{\infty}^{*}$ and $v \in I_{\infty}$.

In the one-weight case the $A_{\infty}$ condition implies the $I_{\infty}$ condition; this gives a result in $\mathbb{R}^{n}$ analogous to Theorem 1.1 .

Theorem 1.7. Given a weight $w$, then for $0<p<\infty$ the strong-type norm inequality

$$
\int_{\mathbb{R}^{n}}\left(M_{0}^{*} f\right)^{p} w d x \leq C \int_{\mathbb{R}^{n}}|f|^{p} w d x
$$

holds for all $f \in L^{p}(w)$ if and only if $w \in A_{\infty}$.

We conclude Section 4 with an example showing that in the two-weight case the $W_{\infty}^{*}$ condition does not imply the $I_{\infty}$ condition. This example has the following interesting consequence: the Sawyer-type condition associated with $M_{0}^{*}$,

$$
\int_{I} M_{0}^{*}\left(v^{-1} \chi_{I}\right) u d x \leq C|I|
$$

while necessary, is not sufficient for the strong-type norm inequality for $M_{0}^{*}$. 
Finally, Section 5 is an appendix which contains a problem about a possible two-weight generalization of the $A_{\infty}$ condition.

Throughout this paper all notation is standard or will be defined as needed. All cubes are assumed to have their sides parallel to the coordinate axes. Given a cube $I, l(I)$ will denote the length of its sides. By weights we will always mean non-negative functions which are locally integrable and positive on a set of positive measure. Given a Borel set $E$ and a weight $v,|E|$ will denote the Lebesgue measure of $E$, $v(E)=\int_{E} v d x$, and $v / \chi_{E}$ will denote the function equal to $v$ on $E$ and infinity elsewhere. Given $1<p<\infty, p^{\prime}=p /(p-1)$ will denote the conjugate exponent of $p$. Finally, $C$ will denote a positive constant whose value may change at each appearance.

\section{Conditions for the Equality of $M_{0} f$ and $M_{0}^{*} f$}

In this section we prove Theorem 1.4. We begin with a simple observation which, since we will use it in later sections, we designate as a lemma.

Lemma 2.1. For all non-negative functions $f$ and all $p>0$, $M_{0}\left(f^{p}\right)=\left(M_{0} f\right)^{p}$ and $M_{0}^{*}\left(f^{p}\right)=\left(M_{0}^{*} f\right)^{p}$.

Proof: For $M_{0}$ this follows immediately from the definition. For $M_{0}^{*}$ the proof is almost as simple: given $x \in \mathbb{R}^{n}$ and $\epsilon>0$, for every $r>0$ there exists a cube $I$ containing $x$ such that

$$
M_{0}^{*}\left(f^{p}\right)(x)-\epsilon \leq\left(\frac{1}{|I|} \int_{I}\left(f^{p}\right)^{r} d x\right)^{1 / r} \leq M_{r p} f(x)^{p}
$$

If we take the limit as $r$ tends to zero we get (since $\epsilon$ is arbitrary) that $M_{0}^{*}\left(f^{p}\right)(x) \leq M_{0}^{*} f(x)^{p}$. An identical argument gives the reverse inequality, and we are done.

Proof of the Sufficiency of Condition (1): Fix a function $f \in L^{p}\left(\mathbb{R}^{n}\right)$ such that $\log |f| \in L_{\text {loc }}^{1}$. Without loss of generality we may assume that $f$ is non-negative. Further, by Lemma 2.1 we may also assume that $p=1$. Now for each $k>0$ define

$$
f_{k}(x)= \begin{cases}f(x) & \text { if } f(x) \geq 1 / k \\ 1 / k & \text { if } f(x)<1 / k\end{cases}
$$


We will first show that $M_{0} f_{k}(x)=M_{0}^{*} f_{k}(x)$ for almost every $x$. Since $k f \in L^{1}$ if $f$ is, and since both $M_{0}$ and $M_{0}^{*}$ are positive homogeneous, we may assume without loss of generality that $k=1$. Furthermore, it will suffice to show that $M_{0}^{*} f_{1}(x) \leq M_{0} f_{1}(x)$ a.e.

Fix $x \in \mathbb{R}^{n}$. There are two cases: If $M_{0}^{*} f_{1}(x)=1$, then by the Lebesgue differentiation theorem (since both $f$ and $\log f$ are locally integrable) for almost every such $x, 1 \leq f_{1}(x) \leq M_{0}^{*} f_{1}(x)$, and so $M_{0} f_{1}(x) \geq f_{1}(x)=1$. If there exists $\epsilon>0$ such that $M_{0}^{*} f_{1}(x)>$ $1+\epsilon$, then for each integer $n>0, M_{1 / n} f_{1}(x) \geq 1+\epsilon$. Define the set $E=\{x: f(x)>1\}$. Then for any $n>0$ and for any cube $I$ such that $\|f\|_{1} /|I|<\epsilon$,

$$
\left(\frac{1}{|I|} \int_{I} f_{1}^{1 / n} d x\right)^{n} \leq \frac{1}{|I|} \int_{I \cap E} f d x+\frac{|I \backslash E|}{|I|} \leq \frac{\|f\|_{1}}{|I|}+1<1+\epsilon .
$$

Hence the cubes used to calculate $M_{1 / n} f_{1}(x)$ must be uniformly bounded in volume. In particular, fix $\delta>0$; then for each $n>0$ there exists a cube $I_{n}$ containing $x$ such that $\left|I_{n}\right|$ is uniformly bounded and

$$
M_{1 / n} f_{1}(x)-\delta<\left(\frac{1}{\left|I_{n}\right|} \int_{I_{n}} f_{1}^{1 / n} d x\right)^{n}
$$

Elementary calculus shows that for all $x \geq 1$ and integers $n>0$, $x^{1 / n} \leq 1+(\log x) / n+x / n^{2}$. Therefore,

$$
\begin{aligned}
M_{1 / n} f_{1}(x)-\delta & <\left(1+\frac{1}{n\left|I_{n}\right|} \int_{I_{n}} \log f_{1} d x+\frac{1}{n^{2}\left|I_{n}\right|} \int_{I_{n}} f_{1} d x\right)^{n} \\
& \leq\left(1+\frac{1}{n\left|I_{n}\right|} \int_{I_{n}} \log f_{1} d x+\frac{1}{n^{2}} M f_{1}(x)\right)^{n} .
\end{aligned}
$$

Since $f \in L^{1}$ and since $M f_{1}(x) \leq M f(x)+1, M f_{1}(x)$ is finite for almost every $x$. Further, since the $I_{n}$ 's are uniformly bounded in size and all contain $x$, by passing to a subsequence we may assume that they converge either to a non-degenerate cube $I$ or to the set $\{x\}$. In the first case

$$
\frac{1}{\left|I_{n}\right|} \int_{I_{n}} \log f_{1} d x \quad \text { converges to } \frac{1}{|I|} \int_{I} \log f_{1} d x
$$

in the second case, by the Lebesgue differentiation theorem it converges to $\log f_{1}(x)$ for almost every $x$. But if a sequence $\left\{a_{n}\right\}$ converges to $a$ and if $M \geq 0$, then

$$
\lim _{n \rightarrow \infty}\left(1+\frac{a_{n}}{n}+\frac{M}{n^{2}}\right)^{n}=e^{a} .
$$


In either case, therefore, if we take the limit in inequality (2) we have that

$$
M_{0}^{*} f_{1}(x)-\delta \leq M_{0} f_{1}(x) \quad \text { a.e. }
$$

Since $\delta>0$ was arbitrary, this establishes the desired inequality.

To complete the proof, since for each $k, M_{0}^{*} f(x) \leq M_{0}^{*} f_{k}(x)=M_{0} f_{k}(x)$ a.e., we only need to show that

$$
\lim _{k \rightarrow \infty} M_{0} f_{k}(x) \leq M_{0} f(x) \quad \text { a.e. }
$$

The argument is similar to the one just given. Fix $x$; since $\log f$ is locally integrable, there exists $\gamma$ such that $M_{0} f(x)=\gamma>0$. Fix $k>2 / \gamma$ and a cube $I$ containing $x$ such that $\|f\|_{1} /|I|<\gamma / 2$. Define $E_{k}=\{x: f \geq 1 / k\}$; then by Jensen's inequality,

$$
\exp \left(\frac{1}{|I|} \int_{I} \log f_{k} d x\right) \leq \frac{1}{|I|} \int_{I} f_{k} d x \leq \frac{1}{|I|} \int_{I \cap E_{k}} f d x+1 / k<\gamma .
$$

Therefore, for each $\delta>0$ there exists a sequence of cubes $I_{k}$ containing $x$ such that $\cup_{k} I_{k}$ is contained in some cube $J$, and such that

$$
\begin{aligned}
M_{0} f_{k}(x)-\delta & <\exp \left(\frac{1}{\left|I_{k}\right|} \int_{I_{k}} \log f_{k} d x\right) \\
& =\exp \left(\frac{1}{\left|I_{k}\right|} \int_{I_{k}} \log f d x+\frac{1}{\left|I_{k}\right|} \int_{I_{k}} \log \left(f_{k} / f\right) d x\right) \\
& \leq M_{0} f(x) \cdot \exp \left[M\left(\log \left(f_{k} / f\right) \chi_{J}\right)(x)\right] .
\end{aligned}
$$

Inequality (3) would follow immediately if we could show that

$$
\lim _{k \rightarrow \infty} M\left(\log \left(f_{k} / f\right) \chi_{J}\right)(x)=0 \quad \text { a.e. }
$$

To show equation (4), first note that

$$
\log \left(f_{k} / f\right)(x)= \begin{cases}0 & \text { if } f(x) \geq 1 / k \\ \log (1 / f)-\log k & \text { if } f(x)<1 / k .\end{cases}
$$

Therefore $0 \leq \log \left(f_{k} / f\right) \leq|\log (1 / f)|$, and so $\log \left(f_{k} / f\right) \in L^{1}(J)$. Since $\log \left(f_{k} / f\right)$ tends to zero pointwise as $k$ tends to infinity, by the dominated convergence theorem it tends to zero in $L^{1}$ norm (on $J$ ). By the weak $(1,1)$ inequality for the Hardy-Littlewood maximal operator, for each $t>0$,

$$
\left|\left\{x \in J: M\left(\log \left(f_{k} / f\right) \chi_{J}\right)(x)>t\right\}\right| \leq \frac{C}{t} \int_{J} \log \left(f_{k} / f\right) d x .
$$


Therefore the sequence $\left\{M\left(\log \left(f_{k} / f\right) \chi_{J}\right)\right\}$ tends to zero in measure, and so has a subsequence which converges to zero pointwise almost everywhere. However, the whole sequence is monotonically decreasing, so in fact (4) holds. This completes the proof of the sufficiency of condition (1).

This proof has the following corollary which we will need below.

Corollary 2.2. Let $I_{0}$ be a cube, and suppose $\operatorname{supp} f=I_{0}$. If for some $p, 0<p<\infty, f \in L^{p}\left(I_{0}\right)$ and $\log f \in L^{1}\left(I_{0}\right)$ then $M_{0}^{*} f(x)=M_{0} f(x)$ for almost every $x$.

Proof: For $x \in I_{0}$ the above proof holds with essentially no modification. For $x$ outside the support of $f$ a direct computation shows that $M_{0} f(x)=M_{0}^{*} f(x)=0$.

Proof of the Sufficiency of Condition (2): Fix $f \in L^{\infty}$; again we may assume that $f$ is non-negative. If $\alpha>2$ then by Hölder's inequality, $M\left(|\log f|^{2}\right)(x) \leq M\left(|\log f|^{\alpha}\right)(x)^{2 / \alpha}<\infty$, so without loss of generality we may assume that $1<\alpha \leq 2$. But then we have the inequality

$$
1+x \leq e^{x} \leq 1+x+|x|^{\alpha}, \quad 0 \leq x \leq 1 .
$$

Let $g(x)=f(x) /\|f\|_{\infty}$. Then for any $n>0$, any $x$ and any cube $I$ containing $x$,

$$
\begin{aligned}
\left(\frac{1}{|I|} \int_{I} g^{1 / n} d x\right)^{n} & =\left(\frac{1}{|I|} \int_{I} e^{(1 / n) \log g} d x\right)^{n} \\
& \leq\left(1+\frac{1}{n|I|} \int_{I} \log g d x+\frac{1}{n^{\alpha}|I|} \int_{I}|\log g|^{\alpha} d x\right)^{n} \\
& \leq M_{0} g(x) \cdot \exp \left(n^{1-\alpha} M\left(|\log g|^{\alpha}\right)(x)\right) .
\end{aligned}
$$

Now for almost every $x$,

$$
M\left(|\log g|^{\alpha}\right)(x) \leq 2^{\alpha} M\left(|\log f|^{\alpha}\right)(x)+2^{\alpha}\left|\log \left(\|f\|_{\infty}\right)\right|<\infty .
$$

Therefore, for each such $x$ we can take the supremum over all $I$ containing $x$ and then the limit as $n$ tends to infinity to get $M_{0}^{*} g(x) \leq M_{0} g(x)$ a.e. Then by homogeneity, $M_{0}^{*} f(x) \leq M_{0} f(x)$ a.e. and we are done. 
Examples. We now give three examples to show that the hypotheses of Theorem 1.4 cannot be weakened in general. For simplicity we construct all the examples on the real line.

First recall the example $f=\chi_{C}, C$ nowhere dense and $|C|=1 / 2$, given in Section 1 above. This shows that $\log f$ needs to be locally integrable.

Example 2.3. There exists a non-negative function $f$ such that $\log f \in L_{\text {loc }}^{1}, f \notin L^{p}(\mathbb{R})$ for any $p, 0<p<\infty$, and such that for all $x, M_{0}^{*} f(x)=\infty$ and $M_{0} f(x)<\infty$.

Proof: Define the function $f$ by

$$
f(x)= \begin{cases}e^{n^{2}} & \text { if }|x| \in\left[e^{n}-1, e^{n}\right], \quad n \geq 1, \\ e^{-1} & \text { otherwise. }\end{cases}
$$

Then $\log f$ is locally integrable but $f \notin L^{p}(\mathbb{R})$ for any finite $p$. Now fix $n$ and let $k>n$. Then

$$
\left(\frac{1}{e^{k}} \int_{0}^{e^{k}} f^{1 / n} d x\right)^{n} \geq\left(e^{k^{2} / n-k}\right)^{n} .
$$

The right-hand side tends to infinity as $k$ tends to infinity. Therefore, for all $x \geq 0, M_{1 / n} f(x)=\infty$, and so $M_{0}^{*} f(x)=\infty$. An identical argument holds for $x<0$.

To see that $M_{0} f$ is everywhere finite, first note that since $\log f$ is locally bounded, given $x \in \mathbb{R}, M_{0} f(x)$ will be infinite only if

$$
\limsup _{\substack{|I| \rightarrow \infty \\ I \ni x}} \frac{1}{|I|} \int_{I} \log f d x=\infty .
$$

Let $x=0$; then

$$
\frac{1}{e^{n}} \int_{0}^{e^{n}} \log f d x=\frac{1}{e^{n}} \sum_{k=1}^{n} k^{2}-\frac{e^{n}-n}{e^{n}}=-1+O\left(n^{3} / e^{n}\right),
$$

and it follows from this that the limit supremum in (5) is finite. A similar argument shows that $M_{0} f(x)<\infty$ for all $x$.

Example 2.4. There exists a function $f \in L^{\infty}(\mathbb{R})$ such that $M(\log f)(x)<\infty$ for all $x$, and for all $x<0, M_{0}^{*} f(x)=2$ and $M_{0} f(x)=1$. 
Proof: For each integer $n \geq 0$, let $a_{n}=2^{-\left(2^{2 n-1}-1\right)}$, and define $f$ by

$$
f(x)= \begin{cases}1 & \text { if } x<0 \\ 2 & \text { if } x \geq 0 \text { and } x \notin\left[2^{n}-1 / 2^{n}, 2^{n}\right], \quad n \geq 0 \\ a_{n} & \text { if } x \in\left[2^{n}-1 / 2^{n}, 2^{n}\right], \quad n \geq 0\end{cases}
$$

Since $\log f$ is locally bounded, to show that $M(\log f)$ is everywhere finite we only need to show that for any $x \in \mathbb{R}$, the limit supremum in (5) is finite. Let $x=0$. Then

$$
\frac{1}{2^{n}} \int_{0}^{2^{n}}|\log f| d x \leq \frac{\log 2}{2^{n}}+\frac{1}{2^{n}} \sum_{k=0}^{n} \frac{2^{2 k-1}-1}{2^{k}} \leq \frac{\log 2}{2^{n}}+1 .
$$

Hence $M(\log f)(0)<\infty$. A similar but lengthier argument shows that $M(\log f)(x)<\infty$ for all $x$.

Now for any $x<0, r>0$ and $n>0$,

$$
\left(\frac{1}{2^{n}-x} \int_{x}^{2^{n}} f^{r} d x\right)^{1 / r} \geq\left(\frac{2^{r}}{2^{n}-x}\left(2^{n}-2+1 / 2^{n}\right)\right)^{1 / r}
$$

The right-hand side tends to 2 as $n$ tends to infinity. Therefore $M_{r} f(x)=$ 2 for all $r>0$, so $M_{0}^{*} f(x)=2$.

Finally, fix $y>0$. Then for some $k \geq 0,2^{k-1}<y \leq 2^{k}-1 / 2^{k}$, or $2^{k}-1 / 2^{k} \leq y \leq 2^{k}$. In either case, by our choice of the $a_{n}$ 's,

$$
\int_{0}^{y} \log f d x \leq \int_{0}^{2^{k}-1 / 2^{k}} \log f d x=0
$$

It follows from this that for all $x<0, M_{0} f(x)=1$.

Finally, note that an estimate similar to the one in Example 2.3 shows that for all $x$ and all $\alpha>1, M\left(|\log f|^{\alpha}\right)(x)=\infty$.

\section{Norm Inequalities for $M_{0}$}

In this section we give new proofs of Theorems 1.1, 1.2 and 1.3. For each theorem we restrict ourselves to proving the sufficiency of the given weight classes: the necessity follows at once if we substitute the test function $v^{-1} \chi_{I}$ into the corresponding norm inequality.

Our proofs depend on the weighted norm inequalities for the minimal operator. 
Theorem 3.1. Given $p>0$ and a pair of weights $(u, v)$ on $\mathbb{R}$, the following are equivalent:

1. $(u, v) \in W_{p}$ : there exists a constant $C$ such that given any interval $I \subset \mathbb{R}$,

$$
\frac{1}{|I|} \int_{I} u d x \leq C\left(\frac{1}{|I|} \int_{I} v^{1 /(p+1)} d x\right)^{p+1}
$$

2. the weak-type inequality

$$
u(\{x: m f(x)<1 / t\}) \leq \frac{C}{t^{p}} \int_{\mathbb{R}} \frac{v}{|f|^{p}} d x
$$

holds for every $f$ such that $1 / f$ is in $L^{p}(v)$;

3. $(u, v) \in W_{p}^{*}$ : there exists a constant $C$ such that given any interval $I \subset \mathbb{R}$,

$$
\int_{I} \frac{u}{m\left(\sigma / \chi_{I}\right)^{p}} d x \leq C \int_{I} \sigma d x
$$

where $\sigma=v^{1 /(p+1)}$;

4. the strong-type inequality

$$
\int_{\mathbb{R}} \frac{u}{(m f)^{p}} d x \leq C \int_{\mathbb{R}} \frac{v}{|f|^{p}} d x
$$

holds for every $f$ such that $1 / f$ is in $L^{p}(v)$.

The constants in (2) and (4) only depend on the constants in (1) and (3) and are independent of $p$.

In the special case where $u=v$ then $W_{p}=W_{p}^{*}=A_{\infty}$ and inequalities (2) and (4) hold in $\mathbb{R}^{n}$ for all $n \geq 1$.

The proof of Theorem 3.1 for equal weights is in Cruz-Uribe and Neugebauer [2]. The two-weight case is in Cruz-Uribe, Neugebauer and Olesen [3].

To make the connection between the minimal operator and the geometric maximal operator, we first define the geometric minimal operator: given a function $f$ on $\mathbb{R}^{n}$, the geometric minimal function of $f$ is

$$
m_{0} f(x)=\inf _{I} \exp \left(\frac{1}{|I|} \int_{I} \log |f| d y\right),
$$

where the infimum is taken over all cubes $I$ containing $x$. It is immediate from this definition that $\left(m_{0} f\right)^{-1}=M_{0}\left(f^{-1}\right)$ for all $f$. Now, as we did 
for the geometric maximal operator, we define a sequence of minimal operators

$$
m_{r} f(x)=\inf _{I}\left(\frac{1}{|I|} \int_{I}|f|^{r} d y\right)^{1 / r}=m\left(f^{r}\right)^{1 / r}
$$

and a limiting minimal operator

$$
m_{0}^{*} f(x)=\lim _{r \rightarrow 0} m_{r} f(x)
$$

(This sequence is decreasing so the limit exists.) In light of the results in Section 2 above, the next result is quite surprising, especially since the proof is so elementary.

Lemma 2. Given a cube $I_{0}$ (possibly infinite), let $f$ be a function on $\mathbb{R}^{n}$ such that for some $r>0, f^{r} \in L_{\mathrm{loc}}^{1}$ on $I_{0}$. Then for all $x$, $m_{0}\left(f / \chi_{I_{0}}\right)(x)=m_{0}^{*}\left(f / \chi_{I_{0}}\right)(x)$.

Proof: Fix $x$. By Jensen's inequality, $m_{0}\left(f / \chi_{I_{0}}\right)(x) \leq m_{0}^{*}\left(f / \chi_{I_{0}}\right)(x)$. To see the reverse inequality, fix $\epsilon>0$. If $x \in I_{0}$ then there exists a cube $I \subset I_{0}$ containing $x$ such that

$$
\begin{aligned}
m_{0}\left(f / \chi_{I_{0}}\right)(x)+\epsilon & >\exp \left(\frac{1}{|I|} \int_{I} \log |f| d y\right) \\
& =\lim _{r \rightarrow 0}\left(\frac{1}{|I|} \int_{I}|f|^{r} d y\right)^{1 / r} \\
& \geq \lim _{r \rightarrow 0} m_{r}\left(f / \chi_{I_{0}}\right)(x) \\
& =m_{0}^{*}\left(f / \chi_{I_{0}}\right)(x) .
\end{aligned}
$$

Since $\epsilon$ was arbitrary, we are done.

Finally, if $x \notin I_{0}$ then both $m_{0}\left(f / \chi_{I_{0}}\right)(x)$ and $m_{0}^{*}\left(f / \chi_{I_{0}}\right)(x)$ are infinite.

An immediate consequence of Lemma 3.2 and the preceding observation is that if $f^{-1}$ is locally integrable then for any cube $I$, the sequence $\left\{m_{r}\left(f^{-1} / \chi_{I}\right)^{-1}\right\}$ increases to $M_{0}\left(f \chi_{I}\right)$ for all $x$.

The weight classes $W_{\infty}$ and $W_{\infty}^{*}$ of Theorems 1.2 and 1.3 are the formal limits of the classes $W_{p}$ and $W_{p}^{*}$ as $p$ tends to infinity. Furthermore, by Jensen's inequality, if the pair $(u, v)$ is in $W_{\infty}$ then it is in $W_{p}$ for all $p>0$ with a constant independent of $p$. Similarly, suppose $(u, v) \in W_{\infty}^{*}$. Then for all cubes $I$ and all $x \in I$, 


$$
\begin{aligned}
M_{0}\left(v^{-1} \chi_{I}\right)(x) & =m_{0}\left(v / \chi_{I}\right)(x)^{-1} \\
& \geq m\left(v^{1 /(p+1)} / \chi_{I}\right)(x)^{-(p+1)} \\
& \geq m\left(v^{1 /(p+1)} / \chi_{I}\right)(x)^{-p}\left(\frac{1}{|I|} \int_{I} v^{1 /(p+1)} d x\right)^{-1} .
\end{aligned}
$$

If we substitute this into the $W_{\infty}^{*}$ condition we see that $(u, v)$ is in $W_{p}^{*}$ for all $p>0$, again with a constant independent of $p$.

The proofs of Theorems 1.1, 1.2 and 1.3 are now straightforward. We will prove Theorem 1.3; the proofs of the other two are identical. By Lemma 2.1 we only need to consider the case $p=1$. Fix $f \in L^{1}(v)$ and for each $n>0$, let $I_{n}=[-n, n]$. Then for each $\epsilon>0,1 /(f+\epsilon)$ is locally integrable. For every $r>0$, since $(u, v) \in W_{\infty}^{*} \subset W_{1 / r}^{*}$, by Theorem 3.1

$$
\int_{\mathbb{R}} \frac{u}{m_{r}\left((f+\epsilon)^{-1} / \chi_{I_{n}}\right)} d x=\int_{\mathbb{R}} \frac{u}{m\left((f+\epsilon)^{-r} / \chi_{I_{n}}\right)^{1 / r}} d x \leq C \int_{I_{n}}(f+\epsilon) v d x .
$$

Since the constant $C$ is independent of $r$, by Lemma 3.2 and the remark following, if we let $r$ tend to 0 , by the monotone convergence theorem we get

$$
\int_{\mathbb{R}} M_{0}\left(f \chi_{I_{n}}\right) u d x \leq \int_{\mathbb{R}} M_{0}\left((f+\epsilon) \chi_{I_{n}}\right) u d x \leq C \int_{I_{n}}(f+\epsilon) v d x .
$$

Since $v$ is locally integrable, the right-hand side is finite, so we can take the limit as $\epsilon$ tends to 0 to get

$$
\int_{\mathbb{R}} M_{0}\left(f \chi_{I_{n}}\right) u d x \leq C \int_{I_{n}} f v d x
$$

Since $M_{0}\left(f \chi_{I_{n}}\right)$ increases to $M_{0} f$, the desired inequality follows from the monotone convergence theorem.

We conclude this section with an example of a pair of weights $(u, v)$ which is in $W_{\infty} \backslash W_{\infty}^{*}$. Our example is simpler than the one given by Yin and Muckenhoupt [13]. Initially we believed that no such example existed, since for all $p>0$ the classes $W_{p}$ and $W_{p}^{*}$ are the same. However, a close examination of the proof that they are the same showed that the constant depended on $p$. Attempts to eliminate this dependency instead yielded the following example. The underlying idea of the construction is to fix an increasing function $v$ which is not a doubling weight and find the "largest" function $u$ such that $(u, v) \in W_{\infty}$.

Example 3.3. There exists a pair of weights $(u, v)$ on $\mathbb{R}$ in $W_{\infty} \backslash W_{\infty}^{*}$. 
Proof: For $x>0$ define the the functions

$$
u(x)=(1+1 / \sqrt{x}) e^{-2 / \sqrt{x}}, \quad v(x)=e^{-1 / \sqrt{x}},
$$

and extend them to $\mathbb{R}$ by making them identically zero for $x \leq 0$. For intervals of the form $I=[-s, t], s \geq 0, t>0$, we have

$$
\frac{1}{|I|} \int_{I} u d x=\frac{t e^{-2 / \sqrt{t}}}{s+t}, \quad \text { and } \quad \exp \left(\frac{1}{|I|} \int_{I} \log v d x\right)=\exp \left(\frac{-2 \sqrt{t}}{s+t}\right) .
$$

Since

$$
\exp \left(\frac{-2 \sqrt{t}}{s+t}+\frac{2}{\sqrt{t}}\right) \geq 1 \geq \frac{t}{s+t}
$$

it follows that $(u, v)$ satisfies the $W_{\infty}$ condition on all such intervals. (Note that when $s=0$ equality holds; it is in this sense that $u$ is the largest possible function.)

Now fix $I=[s, t], 0<s<t$. The $W_{\infty}$ condition follows from the inequality

$$
\frac{t e^{-2 / \sqrt{t}}-s e^{-2 / \sqrt{s}}}{t-s} \leq 2 \exp \left(\frac{-2}{\sqrt{s}+\sqrt{t}}\right)
$$

If $t \geq 2 s$ then this inequality is immediate. Now suppose that $t \leq 2 s$. Since $u$ is an increasing function, the left-hand side of inequality (6) is smaller than $u(t)$. Hence it will suffice to show that

$$
1+1 / \sqrt{t} \leq 2 \exp \left(\frac{2 \sqrt{s}}{\sqrt{t}(\sqrt{s}+\sqrt{t})}\right)
$$

However, since $e^{x} \geq 1+x$,

$2 \exp \left(\frac{2 \sqrt{s}}{\sqrt{t}(\sqrt{s}+\sqrt{t})}\right) \geq 2+\frac{4 \sqrt{s}}{\sqrt{t}(\sqrt{s}+\sqrt{t})} \geq 2+\frac{1-\sqrt{t}}{\sqrt{t}}=1+1 / \sqrt{t}$.

(The last inequality holds since $t \leq 2 s$.) Therefore, $(u, v)$ is in $W_{\infty}$.

To see that $(u, v) \notin W_{\infty}^{*}$, let $I=[0, t], t>0$. Then for all $x \in I$, $M_{0}\left(v^{-1} \chi_{I}\right)(x) \geq e^{2 / \sqrt{x}}$. Therefore,

$$
\frac{1}{|I|} \int_{I} M_{0}\left(v^{-1} \chi_{I}\right) u d x \geq \frac{1}{t} \int_{0}^{t}(1+1 / \sqrt{x}) d x=1+2 / \sqrt{t}
$$

Since the right-hand side tends to infinity as $t$ tends to zero, $(u, v)$ cannot be in $W_{\infty}^{*}$. 


\section{Norm Inequalities for $M_{0}^{*}$}

In this section we prove Theorems 1.5, 1.6 and 1.7. Each of these theorems is a consequence of the corresponding norm inequality for $M_{0}$.

Proof of Sufficiency: We begin with three lemmas which together show that the $I_{\infty}$ condition allows us to reduce the proof to the case of functions of compact support.

Lemma 4.1. Suppose $v \in I_{\infty}$. Then for all $x_{0} \in \mathbb{R}^{n}$,

$$
\limsup _{I, \sigma} \frac{1}{|I|}\left(\frac{1}{|I|} \int_{I} v^{-\sigma} d x\right)^{1 / \sigma}<\infty
$$

where the limit supremum is taken over all cubes $I$ containing $x_{0}$ and $\sigma>0$ as $|I|$ tends to infinity and $\sigma$ tends to zero.

Proof: Suppose to the contrary that there exists an $x_{0}$ such that the given limit supremum is infinite. Then there exists a sequence of cubes $I_{k}$ containing $x_{0}$ such that $\left|I_{k}\right|$ tends to infinity, and a sequence of real numbers $\sigma_{k}$ tending to zero such that

$$
\lim _{k \rightarrow \infty} \frac{1}{\left|I_{k}\right|}\left(\frac{1}{\left|I_{k}\right|} \int_{I_{k}} v^{-\sigma_{k}} d x\right)^{1 / \sigma_{k}}=\infty
$$

By Hölder's inequality we may assume that the $\sigma_{k}$ 's tend to zero as slowly as desired.

Now let $J_{k}$ be the smallest cube containing both $I_{k}$ and the origin. Then $\left|J_{k}\right|=\left(1+\epsilon_{k}\right)^{n}\left|I_{k}\right|$, where

$$
1+\epsilon_{k}=\frac{l\left(J_{k}\right)}{l\left(I_{k}\right)} \leq \frac{l\left(I_{k}\right)+\left|x_{0}\right|}{l\left(I_{k}\right)} .
$$

Hence the $\epsilon_{k}$ 's tend to zero, so by the above observation we may assume that $\sigma_{k} \geq \epsilon_{k}$. But then

$$
\frac{1}{\left|J_{k}\right|}\left(\frac{1}{\left|J_{k}\right|} \int_{J_{k}} v^{-\sigma_{k}} d x\right)^{1 / \sigma_{k}} \geq \frac{1}{\left(1+\epsilon_{k}\right)^{n\left(1+1 / \sigma_{k}\right)}} \frac{1}{\left|I_{k}\right|}\left(\frac{1}{\left|I_{k}\right|} \int_{I_{k}} v^{-\sigma_{k}} d x\right)^{1 / \sigma_{k}} .
$$

Since for all $k,\left(1+\epsilon_{k}\right)^{1 / \sigma_{k}} \leq e$, this implies that $v \notin I_{\infty}$, a contradiction. 
Lemma 4.2. Suppose $f$ is a non-negative function on $\mathbb{R}^{n}$ such that for some $r>0, f^{r} \in L_{\text {loc }}^{1}$, and $K$ is any compact set. If $f_{K}=f \chi_{\mathbb{R}^{n} \backslash K}$, then for each $x_{0} \in \mathbb{R}^{n}$,

$$
\limsup _{I, \sigma}\left(\frac{1}{|I|} \int_{I} f^{\sigma} d x\right)^{1 / \sigma}=\limsup _{I, \sigma}\left(\frac{1}{|I|} \int_{I} f_{K}^{\sigma} d x\right)^{1 / \sigma}
$$

where the limit supremum is taken over all cubes $I$ containing $x_{0}$ and $\sigma>0$ as $|I|$ tends to infinity and $\sigma$ tends to zero.

Proof: The left-hand side of equation (7) is always greater than or equal to the right-hand side, so we only need to prove the reverse inequality. If the left-hand side equals zero there is nothing to prove, so we may assume that it is equal to some $\lambda>0$. Then there exists a sequence of cubes $I_{k}$ containing $x_{0}$ and a sequence of real numbers $\sigma_{k}$ such that $\left|I_{k}\right|$ tends to infinity and $\sigma_{k}$ tends to zero, such that

$$
\lim _{k \rightarrow \infty}\left(\frac{1}{\left|I_{k}\right|} \int_{I_{k}} f^{\sigma_{k}} d x\right)^{1 / \sigma_{k}}=\lambda
$$

Since $\lambda$ is the limit supremum over all such $I$ and $\sigma$, and since by Hölder's inequality the terms on the left-hand side get larger if we make the $\sigma_{k}$ 's larger, this limit will still hold if we replace the $\sigma_{k}$ 's by any larger sequence tending to zero. In particular, we may assume that $1 / \sigma_{k} \leq$ $\left|I_{k}\right|^{1 / 2}$.

Now let $J_{k}=I_{k} \cap K$ and $L_{k}=I_{k} \backslash K$. Then

$$
\begin{aligned}
\left(\frac{1}{\left|I_{k}\right|} \int_{I_{k}} f^{\sigma_{k}} d x\right)^{1 / \sigma_{k}} & =\left(\frac{1}{\left|I_{k}\right|} \int_{J_{k}} f^{\sigma_{k}} d x+\frac{1}{\left|I_{k}\right|} \int_{L_{k}} f^{\sigma_{k}} d x\right)^{1 / \sigma_{k}} \\
& =\left(\frac{1}{\left|I_{k}\right|} \int_{I_{k}} f_{K}^{\sigma_{k}} d x\right)^{1 / \sigma_{k}}\left(1+\frac{\int_{J_{k}} f^{\sigma_{k}} d x}{\int_{L_{k}} f^{\sigma_{k}} d x}\right)^{1 / \sigma_{k}} .
\end{aligned}
$$

Since $K$ is compact and $f^{r} \in L_{\text {loc }}^{1}$, by the dominated convergence theorem,

$$
\lim _{k \rightarrow \infty} \int_{J_{k}} f^{\sigma_{k}} d x \leq|K|
$$


Further, since $\lambda>0$, equation (8) implies that there exists $\tau, 0<\tau<1$, such that for all $k$ sufficiently large

$$
\frac{1}{\left|I_{k}\right|} \int_{I_{k}} f^{\sigma_{k}} d x>\tau
$$

Therefore, again for all $k$ sufficiently large,

$$
\frac{1}{\left|I_{k}\right|} \int_{L_{k}} f^{\sigma_{k}} d x>\tau / 2
$$

SO

$$
1 \leq\left(1+\frac{\int_{J_{k}} f^{\sigma_{k}} d x}{\int_{L_{k}} f^{\sigma_{k}} d x}\right)^{1 / \sigma_{k}} \leq\left(1+\frac{4|K|}{\tau\left|I_{k}\right|}\right)^{1 / \sigma_{k}} \leq\left(1+\frac{4|K|}{\tau\left|I_{k}\right|}\right)^{\left|I_{k}\right|^{1 / 2}}
$$

The right-hand side of this inequality tends to 1 as $k$ tends to infinity. Therefore equations (8) and (9) imply that

$$
\lambda=\limsup _{k \rightarrow \infty}\left(\frac{1}{\left|I_{k}\right|} \int_{I_{k}} f_{K}^{\sigma_{k}} d x\right)^{1 / \sigma_{k}} \leq \limsup _{I, \sigma}\left(\frac{1}{|I|} \int_{I} f_{K}^{\sigma} d x\right)^{1 / \sigma},
$$

and this establishes the desired inequality.

Lemma 4.3. Let $v \in I_{\infty}$ and suppose $f \in L^{1}(v)$. Let $Q_{n}$ be the cube centered at the origin of side-length $2 n$. Then for all $x$,

$$
M_{0}^{*} f(x)=\lim _{n \rightarrow \infty} M_{0}^{*}\left(f \chi_{Q_{n}}\right)(x) .
$$

Proof: Without loss of generality we may assume that $f$ is non-negative. Since $v \in I_{\infty}$, there exists $M>0$ and $\sigma_{0}>0$ such that, given a cube $I$ containing the origin with $|I|>M$, then

$$
\frac{1}{|I|}\left(\frac{1}{|I|} \int_{I} v^{-\sigma_{0}} d x\right)^{1 / \sigma_{0}} \leq C<\infty
$$

Therefore, by Hölder's inequality we have that for all such cubes $I$,

$$
\left(\frac{1}{|I|} \int_{I} f^{\sigma} d x\right)^{1 / \sigma} \leq \int_{\mathbb{R}^{n}} f v d x \cdot \frac{1}{|I|}\left(\frac{1}{|I|} \int_{I} v^{-\sigma /(1-\sigma)} d x\right)^{(1-\sigma) / \sigma}
$$

Since $f \in L^{1}(v), f^{\sigma} \in L_{\text {loc }}^{1}$ provided $\sigma /(1-\sigma) \leq \sigma_{0}$. 
Now fix $x \in \mathbb{R}^{n}$. Suppose first that there exists $N>0$ and a sequence of cubes $I_{k}$ containing $x$ and contained in $Q_{N}$ such that

$$
M_{0}^{*} f(x)=\lim _{k \rightarrow \infty}\left(\frac{1}{\left|I_{k}\right|} \int_{I_{k}} f^{1 / k} d x\right)^{k}
$$

Then for all $n \geq N, M_{0}^{*} f(x)=M_{0}^{*}\left(f \chi_{Q_{n}}\right)(x)$, which establishes equation (10).

If no such sequence of cubes exists, then

$$
M_{0}^{*} f(x)=\limsup _{I, \sigma}\left(\frac{1}{|I|} \int_{I} f^{\sigma} d x\right)^{1 / \sigma}
$$

where the limit supremum is taken over all cubes $I$ containing $x$ and $\sigma>0$ as $|I|$ tends to infinity and $\sigma$ tends to zero. We will show that this implies that $M_{0}^{*} f(x)=0$, which in turn implies that equation (10) holds.

To see this, fix $\epsilon>0$. Then there exists a compact set $K$ such that

$$
\int_{\mathbb{R}^{n} \backslash K} f v d x<\epsilon
$$

Let $f_{K}=f \chi_{\mathbb{R}^{n} \backslash K}$. Then by Lemma 4.2 ,

$$
M_{0}^{*} f(x)=\limsup _{I, \sigma}\left(\frac{1}{|I|} \int_{I} f_{K}^{\sigma} d x\right)^{1 / \sigma}
$$

where the limit supremum is taken over the same $I$ and $\sigma$ as in equation (11). We again apply Hölder's inequality: since $v \in I_{\infty}$, by Lemma 4.1 we have that

$$
M_{0}^{*} f(x) \leq \int_{\mathbb{R}^{n} \backslash K} f v d x \cdot \limsup _{I, \sigma} \frac{1}{|I|}\left(\frac{1}{|I|} \int_{I} v^{-\sigma /(1-\sigma)} d x\right)^{(1-\sigma) / \sigma} \leq C \epsilon
$$

Since $\epsilon$ is arbitrary, $M_{0}^{*} f(x)=0$ and we are done. 
We can now prove Theorems 1.5, 1.6 and 1.7. We will only prove Theorem 1.6; the proofs of the other two are identical. (For Theorem 1.7, we note in passing that if $w \in A_{\infty}$ then $w \in A_{p}$ for all $p$ sufficiently large, which immediately implies that $w \in I_{\infty}$.)

First, by Lemma 4.3 and the monotone convergence theorem, it will suffice to prove Theorem 1.6 for functions $f \in L^{p}(v)$ of compact support. Second, by Lemma 2.1 we may assume that $p=1$. Fix such an $f$ and suppose that supp $f \subset Q_{N}$ for some $N>0$. Define the sequence of functions $\left\{f_{n}\right\}$ by

$$
f_{n}(x)= \begin{cases}f(x) & \text { if } f(x) \geq 1 / n \\ 1 / n & \text { if } x \in Q_{N} \text { and } f(x) \leq 1 / n \\ 0 & \text { otherwise }\end{cases}
$$

As we showed in the proof of Lemma 4.3, there exists $r>0$ such that $f^{r}$ is locally integrable. Therefore, $f_{n}^{r} \in L^{1}\left(Q_{N}\right)$, and $\log f_{n} \in L^{1}\left(Q_{N}\right)$. Therefore, by Corollary 2.2,

$$
M_{0}^{*} f(x) \leq M_{0}^{*} f_{n}(x)=M_{0} f_{n}(x) \quad \text { a.e. }
$$

Since $(u, v) \in W_{\infty}^{*}$, for all $n>0$, by Theorem 1.3

$$
\int_{\mathbb{R}} M_{0}^{*} f u d x \leq \int_{\mathbb{R}} M_{0} f_{n} u d x \leq C \int_{\mathbb{R}} f_{n} v d x
$$

Since $f_{n} \leq f+\frac{1}{n} \chi_{Q_{N}}$ and $v$ is locally integrable, by the dominated convergence theorem we can take the limit as $n$ tends to infinity and get the desired inequality.

Proof of Necessity: The necessity of the $W_{\infty}$ and $W_{\infty}^{*}$ conditions in Theorems 1.5, 1.6 and 1.7 follows from their necessity in the corresponding theorems for $M_{0}$. The necessity of the $I_{\infty}$ condition follows from the next lemma since $u$ is positive on a set of positive measure.

Lemma 4.4. Given a weight $v \notin I_{\infty}$, there exists a function $f \in$ $L^{1}(v)$ such that $M_{0}^{*} f(x)=\infty$ for all $x$. 
Proof: Since $v \notin I_{\infty}$, there exists a sequence of cubes $I_{k}$ containing the origin such that $\left|I_{k}\right|$ tends to infinity, and a sequence of real numbers $\sigma_{k}$ tending to zero such that for all $k$,

$$
\left(\frac{1}{\left|I_{k}\right|} \int_{I_{k}} v^{-\sigma_{k}} d x\right)^{1 / \sigma_{k}}>k^{3}\left|I_{k}\right|
$$

For each $k$ let $a_{k}$ be such that $a_{k}\left|I_{k}\right|=1 / k^{2}$, and define the function $f$ by

$$
f(x)=\sum_{k=1}^{\infty} a_{k} v(x)^{-1} \chi_{I_{k}}(x) .
$$

It is immediate that $f \in L^{1}(v)$. Now fix $x \in \mathbb{R}^{n}$ and let $J_{k}$ be the smallest cube containing $x$ and $I_{k}$. Then, as we showed in Lemma 4.1, $\left|I_{k}\right| /\left|J_{k}\right|$ tends to 1 as $k$ tends to infinity.

Let $r>0$; then for all $k$ such that $\sigma_{k}<r$,

$$
\begin{aligned}
M_{r} f(x) & \geq\left(\frac{1}{\left|J_{k}\right|} \int_{J_{k}} f^{r} d x\right)^{1 / r} \\
& \geq\left(\frac{\left|I_{k}\right|}{\left|J_{k}\right|}\right)^{1 / r}\left(\frac{1}{\left|I_{k}\right|} \int_{I_{k}} f^{\sigma_{k}} d x\right)^{1 / \sigma_{k}} \\
& \geq a_{k}\left(\frac{\left|I_{k}\right|}{\left|J_{k}\right|}\right)^{1 / r}\left(\frac{1}{\left|I_{k}\right|} \int_{I_{k}} v^{-\sigma_{k}} d x\right)^{1 / \sigma_{k}} \\
& \geq a_{k} k^{3}\left|I_{k}\right|\left(\frac{\left|I_{k}\right|}{\left|J_{k}\right|}\right)^{1 / r} \\
& =k\left(\frac{\left|I_{k}\right|}{\left|J_{k}\right|}\right)^{1 / r} .
\end{aligned}
$$

Therefore $M_{r} f(x)=\infty$, so $M_{0}^{*} f(x)=\infty$.

The Independence of $I_{\infty}$ and $W_{\infty}^{*}$. We give an example to show that the $W_{\infty}^{*}$ condition does not imply the $I_{\infty}$ condition. For simplicity we construct our example on $\mathbb{R}$.

Example 4.5. There exists a pair of weights $(u, v) \in W_{\infty}^{*}$ such that $v \notin I_{\infty}$. 
Proof: Define $u(x)=\chi_{[0,1]}(x)$. For $n \geq 1$ let $I_{n}=\left[2^{n}-1 / 2^{n}, 2^{n}\right]$, $I_{0}=\mathbb{R} \backslash \cup_{n} I_{n}$, and $a_{n}=\exp \left[-2^{2 n-1}(n+1) \log 2\right]$. Now define

$$
v(x)=\chi_{I_{0}}(x)+\sum_{n=1}^{\infty} a_{n} \chi_{I_{n}}(x) .
$$

By our choice of the $a_{n}$ 's, if $J_{n}=\left[2^{-n}, 2^{n}\right]$ and $x \in[0,1]$ then a straight-forward induction argument shows that

$$
M_{0}\left(v^{-1} \chi_{J_{n}}\right)(x) \leq \exp \left(\frac{1}{2^{n}} \sum_{k=1}^{n} \log \left(1 / a_{k}\right)\left|I_{k}\right|\right)=2^{n} .
$$

Therefore, if $J$ is an interval such that $2^{n-1}<|J| \leq 2^{n}$ and which intersects $[0,1]$, then

$$
\int_{J} M_{0}\left(v^{-1} \chi_{J}\right) u d x \leq \int_{J_{n+1}} M_{0}\left(v^{-1} \chi_{J_{n+1}}\right) u d x \leq 2^{n+1} \leq 4|J| .
$$

Hence $(u, v) \in W_{\infty}^{*}$.

However, if we let $\sigma=1 / n$, then

$$
\begin{aligned}
\frac{1}{\left|J_{n}\right|}\left(\frac{1}{\left|J_{n}\right|} \int_{J_{n}} v^{-\sigma} d x\right)^{1 / \sigma} & =\frac{1}{\left|J_{n}\right|}\left(\frac{1}{\left|J_{n}\right|} \int_{J_{n}} v^{-1 / n} d x\right)^{n} \\
& \geq \frac{1}{\left|J_{n}\right|}\left(\frac{1}{\left|J_{n}\right|} \sum_{k=1}^{n} a_{k}^{-1 / n}\left|I_{k}\right|\right)^{n} \\
& \geq \frac{1}{\left|J_{n}\right|}\left(\frac{1}{\left|J_{n}\right|} a_{n}^{-1 / n}\left|I_{n}\right|\right)^{n} \\
& =\frac{\exp \left[2^{2 n-1}(n+1) \log 2\right]}{2^{2 n^{2}+2 n+1}} .
\end{aligned}
$$

The right-hand side tends to infinity as $n$ tends to infinity, so $v$ does not satisfy the $I_{\infty}$ condition.

We conclude with the following observation. In this example both $v$ and $v^{-1}$ are locally integrable, so by Corollary 2.2, for any interval $I$, $M_{0}^{*}\left(v^{-1} \chi_{I}\right)=M_{0}\left(v^{-1} \chi_{I}\right)$ a.e. Hence the pair $(u, v)$ satisfies the Sawyertype condition associated with $M_{0}^{*}$, namely,

$$
\int_{I} M_{0}^{*}\left(v^{-1} \chi_{I}\right) u d x \leq C|I|
$$

but the strong-type norm inequality does not hold for $M_{0}^{*}$. Hence this condition is necessary but is not sufficient. 


\section{Appendix: A Two-Weight Generalization of $A_{\infty}$}

As we noted in Section 1, the two-weight reverse Jensen inequality, $W_{\infty}$, does not characterize the union of the two-weight $A_{p}$ classes. Similarly, the stronger $W_{\infty}^{*}$ condition does not characterize this union either. The same example shows this: the pair $\left(e^{|x|}, e^{|2 x|}\right)$ is in $W_{\infty}^{*}$ but is not in any $A_{p}$ class.

However, suppose $(u, v) \in W_{\infty}^{*}$ and $v \in I_{\infty}$. Then by the remarks at the beginning of the proof of Lemma 4.3, for all $p>0$ sufficiently large, $v$ satisfies the Rubio de Francia condition (1) mentioned in Section 1. In other words, for all $p$ sufficiently large, there exists a function $u_{p}$ such that $\left(u_{p}, v\right) \in S_{p} \subset A_{p}$. The function $u_{p}$ need not equal $u$ for any $p$; however, it is natural to ask the following question.

Question 5.1. Is it possible to find functions $u_{p}$ such that $\left(u_{p}, v\right) \in S_{p}$ and the $u_{p}$ 's converge to $u$ (pointwise or as measures) as $p$ tends to infinity?

If this were true it would establish the two conditions $W_{\infty}^{*}$ and $I_{\infty}$ as the "natural" limit of the $A_{p}$ condition and so give a two-weight notion of $A_{\infty}$.

This question arose as the final draft of this paper was being written and we have no conjecture as to its veracity. However, a straightforward calculation does show that $e^{|2 x|} \in I_{\infty}$, and that for the pair $\left(e^{|x|}, e^{|2 x|}\right)$ we may take $u_{p}=e^{|x|} \chi_{\left[-n_{p}, n_{p}\right]}$ for $n_{p}$ sufficiently large.

\section{References}

1. D. Cruz-Uribe, SFO, The minimal operator in $\mathbb{R}^{n}$, Preprint.

2. D. Cruz-Uribe, SFO, and C. J. Neugebauer, The structure of the reverse Hölder classes, Trans. Amer. Math. Soc. 347 (1995), 2941-2960.

3. D. Cruz-Uribe, SFO, C. J. Neugebauer and V. Olesen, Norm inequalities for the minimal and maximal operator, and differentiation of the integral, Publ. Mat. 41 (1997), 577-604.

4. D. Cruz-Uribe, SFO, C. J. Neugebauer and V. Olesen, The one-sided minimal operator and the one-sided reverse Hölder inequality, Studia Math. 116 (1995), 255-270.

5. D. Cruz-Uribe, SFO, C. J. Neugebauer and V. Olesen, Weighted norm inequalities for a family of one-sided minimal operators, Illinois J. Math. 41 (1997), 77-92. 
6. J. García-Cuerva And J. L. Rubio De Francia, "Weighted Norm Inequalities and Related Topics," North Holland Math. Studies 116, North Holland, Amsterdam, 1985.

7. S. V. HrusčEv, A description of weights satisfying the $A_{\infty}$ condition of Muckenhoupt, Proc. Amer. Math. Soc. 90 (1984), 253-257.

8. J. L. Rubio DE Francia, Boundedness of maximal functions and singular integrals in weighted $L^{p}$ spaces, Proc. Amer. Math. Soc. 83 (1981), 673-679.

9. W. Rudin, "Real and Complex Analysis," McGraw-Hill, New York, 1974.

10. C. Sbordone And I. WiK, Maximal functions and related weight classes, Publ. Mat. 38 (1994), 127-155.

11. X. SHI, Two inequalities related to geometric mean operators, J. Zhejiang Teacher's College 1 (1980), 21-25.

12. H. Wei, S. Xianliang And S. QIYU, $A_{\infty}$ condition characterized by maximal geometric mean operator, in "Harmonic Analysis," Proceedings, Tianjin 1988, Lecture Notes in Mathematics 1494, Springer-Verlag, New York, 1991, pp. 68-72.

13. X. Yin And B. Muckenhoupt, Weighted inequalities for the maximal geometric mean operator, Proc. Amer. Math. Soc. 124 (1996), 75-81.

David Cruz-Uribe, SFO:

Department of Mathematics

Trinity College

Hartford, CT 06106-3100

U.S.A.

e-mail: david.cruzuribe@mail.trincoll.edu

\author{
C. J. Neugebauer: \\ Department of Mathematics \\ Purdue University \\ West Lafayette, IN 47907-1395 \\ U.S.A. \\ e-mail: neug@math.purdue.edu
}

Primera versió rebuda el 20 de setembre de 1997, darrera versió rebuda el 20 d'octubre de 1997 